To appear in the International Journal of General Systems

Vol. 00, No. 00, September 2014, 1-13

\title{
Event-triggered Robust Distributed State Estimation for Sensor Networks with State-Dependent Noises
}

\author{
Hongli Dong ${ }^{a, b *}$ Zidong Wang $^{c, d}$ Fuad E. Alsaadi $^{d}$ Bashir Ahmad $^{e}$ \\ ${ }^{a}$ College of Electrical and Information Engineering, Northeast Petroleum University, Daqing \\ 163318, China \\ ${ }^{b}$ Institute for Automatic Control and Complex Systems, University of Duisburg-Essen, Duisburg \\ 47057, Germany \\ ${ }^{c}$ Department of Computer Science, Brunel University, Uxbridge, Middlesex, UB8 3PH, U.K. \\ ${ }^{d}$ Communication Systems and Networks (CSN) Research Group, Faculty of Engineering, King \\ Abdulaziz University, Jeddah 21589, Saudi Arabia. \\ ${ }^{e}$ Department of Mathematics, King Abdulaziz University, Jeddah 21589, Saudi Arabia. \\ (Received 00 Month 201X; final version received 00 Month 201X)
}

\begin{abstract}
This paper is concerned with the event-triggered distributed state estimation problem for a class of uncertain stochastic systems with state-dependent noises and randomly occurring uncertainties (ROUs) over sensor networks. An event-triggered communication scheme is proposed in order to determine whether the measurements on each sensor should be transmitted to the estimators or not. The norm-bounded uncertainty enters into the system in a random way. Through available output measurements from not only the individual sensor but also its neighboring sensors, a sufficient condition is established for the desired distributed estimator to ensure that the estimation error dynamics is exponentially mean-square stable. These conditions are characterized in terms of the feasibility of a set of linear matrix inequalities, and then the explicit expression are given for the distributed estimator gains. Finally, a simulation example is provided to show the effectiveness of the proposed event-triggered distributed state estimation scheme.
\end{abstract}

Keywords: Distributed state estimation; Randomly occurring uncertainties; State-dependent noises; Sensor networks.

\section{Introduction}

In the past decade, wireless sensor networks have attracted an increasing research attention for their successful applications in a variety of areas including environment monitoring, interactive virtual worlds, health care, information collection and warehouse inventory (Bertrand and Moonen, 2010; Cattivelli and Sayed, 2008, 2010). A fundamental collaborative information processing problem with the wireless sensor networks is how to find distributed estimators or filters to obtain the information about the state vectors of the target plants from observations contaminated with external disturbances. Consequently, considerable research attention has been devoted to the theoretical research on the distributed estimation or filtering algorithms that are capable of estimating stationary signals with low-cost and tracking nonstationary processes with reduced-complexity, and a wealth of literature have appeared on this topic, see, e.g., (Ding et al, 2008; Ding et al.,

${ }^{*}$ Corresponding author. Email: shiningdhl@gmail.com 
2012, 2014; Dong et al., 2012, 2013; Huang et al., 2012; Liang et al., 2012; Shen et al., 2010, 2011; Speranzon et al., 2008; Zhang and Yang , 2014) and the references therein. Different from the traditional single node, in the distributed estimation schemes, the local estimators estimate the system state based on the information not only from itself but also from its neighboring sensors according to the given topology. As such, the essential difficulty in designing distributed estimators depends upon how to deal with the complicated coupling issues between one sensor and its neighboring sensors and how to reflect such couplings in the estimator structure specification.

In sensor networks, the limited battery energy, computational power and memory of the sensor nodes are all changeable in a dynamical way. Therefore, parameter uncertainties are ubiquitous when modeling the target plant and the sensor networks. With rapid development of network technologies, the parameter uncertainties may be subject to random changes and may occur in a probabilistic way, for instance, random network-induced structural changes, repairs of components, changing subsystem interconnections or sudden environment changes, etc. In this sense, it would make practical sense to consider the randomly occurring uncertainties when designing the desired distributed estimation algorithm. Very recently, some pioneering work has appeared in the literature concerning the state estimation problem for a class of discrete nonlinear systems with randomly occurring uncertainties (ROUs), see (Hu et al., 2014). On the other hand, the stochastic disturbances are usually encountered in sensor networks within a noisy environment. Note that many plants may be modeled by systems with state-dependent noises and some characteristics of nonlinear systems can be closely approximated by models with statedependent noises rather than by linearized models (Ding, 2013; Hu et al., 2013; Wang et al., 2010, 2013). Unfortunately, up to now, very little research effort has been paid to the robust distributed estimation issue with simultaneous presence of parameter uncertainties and state-dependent noises.

In the past decades, the event-triggered communication mechanism has received much research attention due to the rapid development of digital microprocessor and computer science. In comparison with conventional time-triggered communication, event-triggering allows a considerable reduction of the network resource occupancy while maintaining the guaranteed filtering performance, avoids some injurious transmission phenomena such as data dropouts and time delay, etc, and extends the lifetime of the services. Therefore, the event-triggered communication mechanism is particularly significant in sensor networks due to its capability of decreasing the unnecessary executions of the systems and saving energy. In the past few years, the event-based strategies have been extensively studied for various engineering systems such as networked control systems (Donkers and Heemels, 2012; Hu and Yue, 2012; Liu et al., 2014; Peng and Yang, 2013), sensor networks (Lee and Choi, 2013; Tseng, 2005), multi-agent systems (Tabuada, 2007; Zhu et al., 2014) and neural networks (Li, 2012; Sahoo et al., 2013), etc. However, the available results in the literature have been scattered for the filtering or state estimation problems, most of which have been concerned with the implementation problems rather than the system analysis and synthesis issues.

Summarizing the above discussion, in this paper, we are motivated to study the robust distributed state estimation problem for a class of uncertain stochastic systems with statedependent noises and randomly occurring uncertainties over the sensor network characterized by a directed graph. By augmenting the state of the original system and the estimation errors on all sensor nodes, sufficient conditions are established for the existence of the desired estimators and then a parameterization of the estimator gains is derived. A simulation example is provided to show the effectiveness of the proposed distributed state estimation scheme. The main contribution of this paper is twofold. 1) A comprehensive model is established over the sensor network which covers event-triggered measurement transmissions, randomly occurring uncertainties (ROUs) and state-dependent noises. 2) 
Intensive stochastic analysis and Kronecker product are conducted to ensure the stability requirement for the addressed "complex" systems.

The rest of this paper is outlined as follows. In Section 2, the discrete-time dynamic plant with a network of $n$ sensors is introduced and the problem under consideration is formulated. In Section 3, the distributed estimator design problem is solved by employing the semi-definite programme method. A simulation example is given in Section 4 to demonstrate the main results obtained. Finally, we conclude the paper in Section 5.

Notation. The notation used here is standard except where otherwise stated. $\mathbb{R}^{n}$ and $\mathbb{R}^{n \times m}$ denote, respectively, the $n$-dimensional Euclidean space and the set of all $n \times m$ real matrices. $\mathbb{N}^{0}$ is used to be describe the set $\{0,1, \ldots\}$. The set of all positive integers is denoted by $\mathbb{I}^{+} . l_{2}[0, \infty)$ is the space of square summable sequences. The notation $X \geq Y$ (respectively, $X>Y$ ), where $X$ and $Y$ are real symmetric matrices, means that $X-Y$ is positive semi-definite (respectively, positive definite). $M^{T}$ represents the transpose of the matrix $M .0$ represents zero matrix of compatible dimensions. The $n$-dimensional identity matrix is denoted as $I_{n}$ or simply $I$, if no confusion is caused. $\operatorname{diag}\{\cdots\}$ stands for a blockdiagonal matrix. $(\Omega, \mathscr{F}$, Prob) is a complete probability space with the probability measure Prob having total mass 1 . Prob $\{\beta\}$ stands for the occurrence probability of the event $\beta$ and $\mathbb{E}\left\{\alpha_{1}\right\}, \mathbb{E}\left\{\alpha_{1} \mid \alpha_{2}\right\}$ mean, respectively, the mathematical expectation of the stochastic variable $\alpha_{1}$ and the expectation of $\alpha_{1}$ conditional on $\alpha_{2}$ with respect to the given probability measure Prob. In symmetric block matrices, "*” is used as an ellipsis for terms induced by symmetry. The symbol $\otimes$ denotes the Kronecker product. $\mathbf{1}_{n}:=[1,1, \ldots, 1]^{T} \in \mathbb{R}^{n}$. Matrices, if they are not explicitly specified, are assumed to have compatible dimensions.

\section{Problem Formulation}

In this paper, we assume that the $n$ sensor nodes are distributed in space according to a fixed network topology represented by a directed graph $\mathcal{G}=(\mathcal{V}, \mathcal{E}, Q)$ of order $n$ with the set of nodes $\mathcal{V}=1,2, \ldots, n$, the set of edges $\mathcal{E} \in \mathcal{V} \times \mathcal{V}$, and the weighted adjacency matrix $Q=\left[a_{i j}\right]$ with nonnegative adjacency element $a_{i j}$. An edge of $\mathcal{G}$ is denoted by ordered pair $(i, j)$. The adjacency elements associated with the edges of the graph are positive, i.e., $a_{i j}>0 \Longleftrightarrow(i, j) \in \mathcal{E}$ which means that sensor $i$ can obtain information from sensor $j$. Also, we assume that $a_{i i}=1$ for all $i \in \mathcal{V}$, and therefore $(i, i)$ can be regarded as an additional edge. The set of neighbors of node $i \in \mathcal{V}$ plus the node itself are denoted by $\mathcal{N}_{i}=\{j \in \mathcal{V}:(i, j) \in \mathcal{E}\}$.

Consider the following class of discrete-time stochastic uncertain systems defined on the complete probability space $(\Omega, \mathscr{F}$, Prob):

$$
x(k+1)=\left(A+\alpha(k) \Delta A+A_{\xi} \xi(k)\right) x(k)+E w(k)
$$

where $x(k) \in \mathbb{R}^{n_{x}}$ is the system state; $w(k) \in \mathbb{R}^{n_{v}}$ is the disturbance input belonging to $l_{2}[0, \infty) ; \xi(k) \in \mathbb{R}$ is a zero mean Gaussian white noise sequence with $\mathbb{E}\left\{\xi^{T}(k) \xi(k)\right\}=1$.

The real-valued matrix $\Delta A$ represents the norm-bounded parameter uncertainty of the following structure

$$
\Delta A=H_{a} F(k) N,
$$

where $H_{a}$ and $N$ are known real constant matrices and $F(k)$ is an unknown matrix function satisfying the following condition

$$
F^{T}(k) F(k) \leq I .
$$


The stochastic variable $\alpha(k) \in \mathbb{R}$ in (1), which characterizes the phenomenon of randomly occurring uncertainties, is a Bernoulli distributed white sequence taking values on either 0 or 1 with

$$
\operatorname{Prob}\{\alpha(k)=1\}=\bar{\alpha}, \quad \operatorname{Prob}\{\alpha(k)=0\}=1-\bar{\alpha},
$$

where $\bar{\alpha} \in[0,1]$ is a known constant, the variables $\xi(k)$ and $\alpha(k)$ are mutually independent.

For the $i$ th sensor, the measurement output is described by

$$
y_{i}(k)=C_{i} x(k)+D_{i} v(k), \quad i=1,2, \ldots, n
$$

and $y_{i}(k) \in \mathbb{R}^{n_{y}}$ is the output measured by sensor $i$ from the plant, and $v(k) \in l_{2}[0, \infty)$ is an external disturbance. Moreover, all the matrices mentioned above, i.e., $A, A_{\xi}, E, C_{i}$ and $D_{i}$, are known matrices with appropriate dimensions.

For the purpose of reducing data communication frequency, the event generator function $f_{i}(\cdot, \cdot)(i=1,2, \ldots, n)$ is constructed as follows:

$$
f_{i}\left(\varphi_{i}(k), \delta_{i}\right)=\varphi_{i}^{T}(k) \Omega_{i} \varphi_{i}(k)-\delta_{i} r_{i}^{T}(k) \Omega_{i} r_{i}(k)
$$

where $r_{i}(k)=y_{i}(k)-C_{i} \hat{x}_{i}(k)$ is the innovation sequence exchanged via the network, $\hat{x}_{i}(k) \in$ $\mathbb{R}^{n_{x}}$ is the estimation of the plant state in the $i$ th sensor node. $\varphi_{i}(k)=r_{i}\left(k_{k}^{j}\right)-r_{i}(k), r_{i}\left(k_{k}^{j}\right)$ is the broadcast innovation at latest event time, $\Omega_{i}(i=1,2, \ldots, n)$ are symmetric positive definite matrices, and $\delta_{i} \in[0,1)$.

The execution is triggered as long as the condition

$$
f_{i}\left(\varphi_{i}(k), \delta_{i}\right)>0
$$

is satisfied. Therefore, the sequence of event-triggered instants $0 \leq k_{0}^{i} \leq k_{1}^{i} \leq \cdots \leq k_{k}^{i} \leq$ $\cdots$ is determined iteratively by

$$
k_{k+1}^{i}=\inf \left\{k \in \mathbb{N}^{0} \mid k>k_{k}^{i}, f_{i}\left(\varphi_{i}(k), \delta_{i}\right)>0\right\} .
$$

In this paper, the following event-triggered distributed state estimator structure is adopted on sensor node $i$ :

$$
\hat{x}_{i}(k+1)=A \hat{x}_{i}(k)+\sum_{j \in \mathcal{N}_{i}} a_{i j} K_{i j} r_{j}\left(k_{k}^{j}\right)
$$

where $K_{i j}$ are the estimator gain matrices on node $i$ to be designed.

For convenience of later analysis, we denote

$$
\begin{aligned}
& e_{i}(k)=x(k)-\hat{x}_{i}(k), \quad \Psi(k)=\left[\varphi_{1}^{T}(k) \varphi_{2}^{T}(k) \cdots \varphi_{n}^{T}(k)\right]^{T}, \\
& e(k)=\left[e_{1}^{T}(k) e_{2}^{T}(k) \cdots e_{n}^{T}(k)\right]^{T}, \quad \bar{x}(k)=\mathbf{1}_{n} \otimes x(k), \\
& \bar{C}=\operatorname{diag}\left\{C_{1}, C_{2}, \ldots, C_{n}\right\}, \quad \bar{D}=\left[\begin{array}{ll}
D_{1}^{T} D_{2}^{T} \cdots D_{n}^{T}
\end{array}\right]^{T}, \\
& \bar{A}=I_{n} \otimes A, \quad \Delta \bar{A}=I_{n} \otimes \Delta A, \quad \Theta=\operatorname{diag}\left\{\delta_{1} I, \delta_{2} I, \ldots, \delta_{n} I\right\}, \\
& \bar{E}=\mathbf{1}_{n} \otimes E, \quad \tilde{\alpha}(k)=\alpha(k)-\bar{\alpha}, \quad \Omega=\operatorname{diag}\left\{\Omega_{1} I, \Omega_{2} I, \ldots, \Omega_{n} I\right\}, \\
& \bar{A}_{\xi}=I_{n} \otimes A_{\xi}
\end{aligned}
$$


where

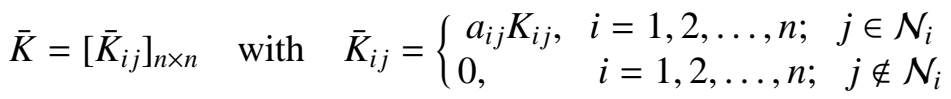

Obviously, since $a_{i j}=0$ when $j \notin \mathcal{N}_{i}, \bar{K}$ is a matrix that can be expressed as

$$
\bar{K} \in \mathscr{T}_{n_{x} \times n_{y}}
$$

where $\mathscr{T}_{p \times q}=\left\{\bar{U}=\left[U_{i j}\right] \in \mathbb{R}^{n p \times n q} \mid U_{i j} \in \mathbb{R}^{p \times q}, U_{i j}=0\right.$ if $\left.j \notin \mathcal{N}_{i}\right\}$.

Letting $\eta(k)=\left[\begin{array}{lll}\bar{x}^{T}(k) & e^{T}(k)\end{array}\right]^{T}$ and $\varpi(k)=\left[\begin{array}{ll}w^{T}(k) & v^{T}(k)\end{array}\right]^{T}$, the following system is obtained that governs the estimator dynamics for the sensor network:

$$
\eta(k+1)=(\mathcal{A}+\bar{\alpha} \Delta \mathcal{A}) \eta(k)+\left(\tilde{\alpha}(k) \Delta \mathcal{A}+\xi(k) \mathcal{A}_{\xi}\right) \eta(k)-\tilde{K} \mathcal{H} \Psi(k)+\mathcal{D} \varpi(k)
$$

where

$$
\begin{aligned}
& \mathcal{A}=\left[\begin{array}{cc}
\bar{A} & 0 \\
0 & \bar{A}-\bar{K} \bar{C}
\end{array}\right], \quad \Delta \mathcal{A}=\left[\begin{array}{cc}
\Delta \bar{A} & 0 \\
\Delta \bar{A} & 0
\end{array}\right], \quad \mathcal{A}_{\xi}=\left[\begin{array}{cc}
\bar{A}_{\xi} & 0 \\
\bar{A}_{\xi} & 0
\end{array}\right], \\
& \mathcal{D}=\left[\begin{array}{cc}
\bar{E} & 0 \\
\bar{E} & -\bar{K} \bar{D}
\end{array}\right], \quad \mathcal{H}=\left[\begin{array}{ll}
0 & I
\end{array}\right]^{T}, \quad \tilde{K}=I_{2} \otimes \bar{K} .
\end{aligned}
$$

Before proceeding further, we introduce the following definition.

Definition 1. The augmented system in (13) is said to be exponentially mean-square stable if, with $\varpi(k)=0$, there exist constants $\varsigma>0$ and $0<\kappa<1$ such that

$$
\mathbb{E}\left\{\|\eta(k)\|^{2}\right\} \leq \varsigma \kappa^{k} \mathbb{E}\left\{\|\eta(0)\|^{2}\right\}, \quad \forall \eta(0) \in \mathbb{R}^{n}, \quad k \in \mathbb{I}^{+}
$$

Our aim in this paper is to design event-triggered distributed state estimators of the form in (9) on each node $i$ of the sensor network for system (1). In other words, we are going to find the distributed state estimator parameters $K_{i j}$ such that the estimation error systems (13) to be exponentially mean-square stable for all randomly occurring parameter uncertainties and state-dependent noises.

\section{Main Results}

In this section, let us investigate the distributed state estimation for system (1) with $n$ sensors whose topology is determined by the given graph $\mathcal{G}=(\mathcal{V}, \mathcal{E}, Q)$. The following lemmas will be needed in establishing our main results.

Lemma 1. (Shen et al., 2010) Let $P=\operatorname{diag}\left\{P_{1}, P_{2}, \ldots, P_{n}\right\}$ with $P_{i} \in \mathbb{R}^{p \times p}(1 \leq i \leq n)$ being invertible matrices. If $X=P W$ for $W \in \mathbb{R}^{n p \times n q}$, then we have $W \in \mathscr{T}_{p \times q} \Longleftrightarrow X \in$ $\mathscr{T}_{p \times q}$.

Lemma 2. (S-procedure) Let $L=L^{\mathrm{T}}$ and $H$ and $E$ be real matrices of appropriate dimensions with $F$ satisfying $F F^{T} \leq I$, then $L+H F E+E^{\mathrm{T}} F^{\mathrm{T}} H^{\mathrm{T}}<0$, if and only if there 
exists a positive scalar $\varepsilon>0$ such that $L+\varepsilon^{-1} H H^{\mathrm{T}}+\varepsilon E^{\mathrm{T}} E<0$ or equivalently,

$$
\left[\begin{array}{ccc}
L & H & \varepsilon E^{\mathrm{T}} \\
H^{\mathrm{T}} & -\varepsilon I & 0 \\
\varepsilon E & 0 & -\varepsilon I
\end{array}\right]<0
$$

The following theorem gives a sufficient condition under which the augmented error system in (13) is exponentially mean-square stable in the sense of Definition 1.

Theorem 1. Consider the discrete-time stochastic uncertain system (1) and sensors (5) with event generator function (6). For the given estimator parameter $\bar{K}$, the augmented estimation error system in (13) is exponentially mean-square stable if there exist a positive definite matrix $P>0$ and a scalar $\lambda>0$ satisfying

$$
\bar{\Gamma}=\left[\begin{array}{cc}
\bar{\Upsilon}_{11} & * \\
-(\tilde{K} \mathcal{H})^{T} P(\mathcal{A}+\bar{\alpha} \Delta \mathcal{A})(\tilde{K} \mathcal{H})^{T} P(\tilde{K} \mathcal{H})-\lambda \Omega
\end{array}\right]<0
$$

where

$$
\begin{aligned}
\bar{\Upsilon}_{11} & =(\mathcal{A}+\bar{\alpha} \Delta \mathcal{A})^{T} P(\mathcal{A}+\bar{\alpha} \Delta \mathcal{A})+g \Delta \mathcal{A}^{T} P \Delta \mathcal{A}-P+\lambda C^{T} \Theta \Omega C+\mathcal{A}_{\xi}^{T} P \mathcal{A}_{\xi}, \\
g & =\bar{\alpha}(1-\bar{\alpha}), C=[0 \bar{C}]
\end{aligned}
$$

Proof. Choose the following Lyapunov function for system (13):

$$
V(\eta(k)):=\eta^{T}(k) P \eta(k)
$$

The difference of the Lyapunov function is given as follows:

$$
\Delta V(\eta(k))=\mathbb{E}\{V(\eta(k+1)) \mid \eta(k)\}-V(\eta(k)) .
$$

Calculating the difference of $V(\eta(k))$ along the trajectory of system (13) with $\varpi(k)=0$ and taking the mathematical expectation, we have

$$
\begin{aligned}
\mathbb{E}\{\Delta V(\eta(k))\}:= & \mathbb{E}\left\{\eta^{T}(k+1) P \eta(k+1)-\eta^{T}(k) P \eta(k)\right\} \\
= & \mathbb{E}\left\{((\mathcal{A}+\bar{\alpha} \Delta \mathcal{A}) \eta(k)+(\tilde{\alpha}(k) \Delta \mathcal{A}+\xi(k) \mathcal{A} \xi) \eta(k)-\tilde{K} \mathcal{H} \Psi(k))^{T} P((\mathcal{A}+\bar{\alpha}\right. \\
& \left.\left.\times \Delta \mathcal{A}) \eta(k)+\left(\tilde{\alpha}(k) \Delta \mathcal{A}+\xi(k) \mathcal{A}_{\xi}\right) \eta(k)-\tilde{K} \mathcal{H} \Psi(k)\right)-\eta^{T}(k) P \eta(k)\right\}
\end{aligned}
$$

In addition, it follows from the event-triggering condition (7) that

$$
\Psi^{T}(k) \Omega \Psi(k)-\eta^{T}(k) C^{T} \Theta \Omega C \eta(k) \leq 0
$$


which results in

$$
\begin{aligned}
\mathbb{E}\{\Delta V(\eta(k))\} \leq & \mathbb{E}\left\{((\mathcal{A}+\bar{\alpha} \Delta \mathcal{A}) \eta(k)+(\tilde{\alpha}(k) \Delta \mathcal{A}+\xi(k) \mathcal{A} \xi) \eta(k)-\tilde{K} \mathcal{H} \Psi(k))^{T} P((\mathcal{A}+\bar{\alpha}\right. \\
& \left.\times \Delta \mathcal{A}) \eta(k)+\left(\tilde{\alpha}(k) \Delta \mathcal{A}+\xi(k) \mathcal{A}_{\xi}\right) \eta(k)-\tilde{K} \mathcal{H} \Psi(k)\right)-\eta^{T}(k) P \eta(k) \\
& \left.-\lambda \Psi^{T}(k) \Omega \Psi(k)+\lambda \eta^{T}(k) C^{T} \Theta \Omega C \eta(k)\right\} \\
= & \mathbb{E}\left\{\eta^{* T}(k) \bar{\Gamma} \eta^{*}(k)\right\}
\end{aligned}
$$

where

$$
\eta^{*}(k):=\left[\eta^{T}(k) \Psi^{T}(k)\right]^{T}
$$

It follows from Theorem 1 that

$$
\mathbb{E}\left\{\Delta V(\eta(k)\} \leq-\lambda_{\min }(-\bar{\Gamma})\left\|\eta^{*}(k)\right\|^{2} .\right.
$$

Finally, we can confirm from Lemma 1 of (Wang et al., 2006) that the augmented estimation error system (13) is exponentially mean-square stable, and the proof is now complete.

After conducting the dynamic analysis in Theorem 1 for the augmented estimation error system (13), we are now in a position to deal with the problem of designing distributed state estimator (9). The solution to the distributed state estimating problem with both randomly occurring uncertainties and state-dependent noises is obtained by the following theorem.

Theorem 2. For the discrete-time stochastic uncertain system (1) and sensors (5) with event generator function (6), the dynamics of estimation error (13) is exponentially meansquare stable if there exist positive constant scalars $\lambda$ and $\varepsilon$, a positive definite matrix $P>0$ and the matrix $\mathcal{K} \in \mathscr{T}_{2 n_{x} \times n_{y}}$ satisfying

$$
\begin{gathered}
P=\operatorname{diag}\left\{P_{1}, P_{2}, \ldots, P_{n}\right\}>0, \\
{\left[\begin{array}{ccc}
\Pi_{11} & * & * \\
\Pi_{21} & -\bar{P} & * \\
\Pi_{31} & \Pi_{32} & \Pi_{33}
\end{array}\right]<0,}
\end{gathered}
$$

where

$$
\begin{aligned}
& \Pi_{11}=\operatorname{diag}\left\{\lambda C^{T} \Theta \Omega C-P,-\lambda \Omega\right\}, \quad \bar{P}=\operatorname{diag}\{P, P, P\}, \\
& \Pi_{21}=\left[\begin{array}{cc}
P \hat{\mathcal{A}}_{0}+\mathcal{K} \hat{\mathcal{C}}_{0}-\mathcal{K} \\
0 & 0 \\
P \mathcal{A}_{\xi} & 0
\end{array}\right], \quad \Pi_{31}=\left[\begin{array}{cc}
0 & 0 \\
\varepsilon \hat{N}_{a} & 0
\end{array}\right], \\
& \Pi_{32}=\left[\begin{array}{ccc}
\bar{\alpha} \hat{H}_{a}^{T} P & \sqrt{g} \hat{H}_{a}^{T} P & 0 \\
0 & 0 & 0
\end{array}\right], \quad \Pi_{33}=-I_{2} \otimes \varepsilon I, \quad \hat{\mathcal{A}}_{0}=I_{2} \otimes \bar{A}, \\
& \hat{\mathcal{C}}_{0}=[0-\bar{C}], \quad \hat{H}_{a}=\mathbf{1}_{2} \otimes\left[I_{n} \otimes H_{a} 0\right], \quad \hat{N}_{a}=\mathbf{1}_{2} \otimes\left[I_{n} \otimes N 0\right]
\end{aligned}
$$


and the other parameters are defined in (10). Moreover, if the above inequalities are feasible, then the matrix $\bar{K}$ is given as follows:

$$
\bar{K}=\left(\mathcal{H}^{T} P \mathcal{H}\right)^{-1} \mathcal{H}^{T} \mathcal{K}
$$

Accordingly, the desired estimator gain parameter $K_{i j}\left(i=1,2, \ldots, n, j \in \mathcal{N}_{i}\right)$ can be obtained from (11).

Proof. It is observed that

$$
\mathcal{A}=\hat{\mathcal{A}}_{0}+\tilde{K} \mathcal{H} \hat{C}_{0}, \quad \Delta \mathcal{A}=\hat{H}_{a} \hat{F}(k) \hat{N}_{a} .
$$

where

$$
\hat{F}(k)=I_{2 n} \otimes F(k)
$$

By applying the Schur Complement Lemma (Boyd et al., 1994) and noting $P \tilde{K} \mathcal{H}=\mathcal{K}$, (16) can be rewritten as

$$
\bar{\Pi}=\left[\begin{array}{cc}
\Pi_{11} & * \\
\bar{\Pi}_{21} & -\bar{P}
\end{array}\right]<0,
$$

where

$$
\bar{\Pi}_{21}=\left[\begin{array}{cc}
P \hat{\mathcal{A}}_{0}+\mathcal{K} \hat{\mathcal{C}}_{0}+\bar{\alpha} P \hat{H}_{a} \hat{F}(k) \hat{N}_{a}-\mathcal{K} \\
\sqrt{g} P \hat{H}_{a} \hat{F}(k) \hat{N}_{a} & 0 \\
P \mathcal{A}_{\xi} & 0
\end{array}\right] .
$$

Furthermore, considering the uncertain parameter $\Delta \mathcal{A}$, we reorganize (27) in terms of Lemma 2 as follows:

$$
\bar{\Pi}=\left[\begin{array}{cc}
\Pi_{11} & * \\
\Pi_{21} & -\bar{P}
\end{array}\right]+\bar{H}_{a} \hat{F}(k) \bar{N}_{a}+\bar{N}_{a}^{T} \hat{F}^{T}(k) \bar{H}_{a}^{T}<0,
$$

where

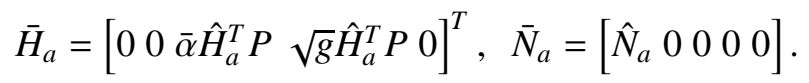

From Lemma 2, we can easily obtain (22). In addition, noting $P(k)=$ $\operatorname{diag}\left\{P_{1}(k), P_{2}(k), \ldots, P_{n}(k)\right\}$, from Lemma 1 , it is easy to verify that the condition $\bar{K} \in$ $\mathscr{T}_{n_{x} \times n_{y}}$ is satisfied. The proof of this theorem is now complete.

Remark 1. It is well known that the main difficulties in designing distributed estimators in sensor networks lie in the tight coupling among sensors in terms of both time and space. In this paper, the estimator parameters $K_{i j}\left(i=1,2, \ldots, n, j \in \mathcal{N}_{i}\right)$ are "assembled" to matrix $\bar{K}$ which should meet the constraint (11). Then, by Lemma 1, we can derive the condition that $\bar{K} \in \mathscr{T}_{n_{x} \times n_{y}}$ is required to satisfy. Consequently, the distributed estimators can be designed effectively. 


\section{An Illustrative Example}

In this section, we present a simulation example to illustrate the effectiveness of the proposed robust event-triggered distributed estimator design scheme for the discrete-time stochastic system with state-dependent noises and randomly occurring uncertainties over sensor networks.

The sensor network is represented by a directed graph $\mathcal{G}=$ $(\mathcal{V}, \mathcal{E}, Q)$ with the set of nodes $\mathcal{V}=\{1,2,3,4\}$, set of edges $\mathcal{E}=$ $\{(1,1),(1,2),(2,2),(2,3),(3,2),(3,3),(3,4),(4,1),(4,4)\}$ and the following adjacency matrix:

$$
\mathcal{A}=\left[\begin{array}{llll}
1 & 1 & 0 & 0 \\
0 & 1 & 1 & 0 \\
0 & 1 & 1 & 1 \\
1 & 0 & 0 & 1
\end{array}\right] .
$$

The system data are given as follows:

$$
\begin{aligned}
& A=\left[\begin{array}{ccc}
0.4 & 0.1 & 0 \\
0 & -0.8 & -0.6 \\
0.1 & 0.2 & -0.5
\end{array}\right], A_{\xi}=\left[\begin{array}{ccc}
0.1 & 0.1 & 0 \\
0 & -0.1 & 0 \\
0 & 0.2 & -0.1
\end{array}\right] E=\left[\begin{array}{lll}
0.5 & 1 & 0.1
\end{array}\right]^{T}, F(k)=\sin (0.6 k), \\
& H_{a}=\left[\begin{array}{lll}
0.1 & 0.2 & 0.1
\end{array}\right]^{T}, N=\left[\begin{array}{lll}
0.2 & 0.1 & 0.2
\end{array}\right], C_{1}=\left[\begin{array}{lll}
0.1 & 0 & 0.1
\end{array}\right], C_{2}=\left[\begin{array}{lll}
0.2 & 0.1 & 0.2
\end{array}\right], \\
& C_{3}=\left[\begin{array}{lll}
0.5 & 0.7 & 0.2
\end{array}\right], C_{4}=\left[\begin{array}{lll}
0.1 & 0.2 & 0.1
\end{array}\right], D_{1}=0.1, D_{2}=0.1, D_{3}=0.2, D_{4}=0.2 \text {. }
\end{aligned}
$$

In this example, the probability of the randomly occurring uncertainty is taken as $\bar{\alpha}=$ 0.8. Choose event weighted matrix $\Omega_{1}=\Omega_{2}=\Omega_{3}=\Omega_{4}=I$ and the threshold $\delta_{1}=\delta_{2}=$ $\delta_{3}=\delta_{4}=0.7$. By solving (22) in Theorem 2, we can obtain the following parameters of the desired distributed estimators:

$$
\begin{array}{ll}
K_{11}=\left[\begin{array}{lll}
0 & 0 & 0
\end{array}\right]^{T}, & K_{12}=\left[\begin{array}{lll}
0.0613 & -0.0222 & 0.0285
\end{array}\right]^{T}, \\
K_{22}=\left[\begin{array}{lll}
0.1632 & -0.1929 & 0.0784
\end{array}\right]^{T}, & K_{23}=\left[\begin{array}{lll}
0.0764 & 0.0543 & 0.0034
\end{array}\right]^{T}, \\
K_{32}=\left[\begin{array}{lll}
0 & 0 & 0
\end{array}\right]^{T}, \quad K_{33}=\left[\begin{array}{lll}
0 & 0 & 0
\end{array}\right]^{T}, & K_{34}=\left[\begin{array}{lll}
0.2652 & -0.1866 & 0.1991
\end{array}\right]^{T}, \\
K_{41}=\left[\begin{array}{lll}
0.1878 & -0.0751 & 0.1736
\end{array}\right]^{T}, & K_{44}=\left[\begin{array}{lll}
0.1330 & -0.1307 & 0.1382
\end{array}\right]^{T}
\end{array}
$$

In the simulation, the exogenous disturbance inputs are selected as $w(k)=$ $\exp (-0.2 k) \sin (k)$ and $v(k)=\frac{\sin (10 k+1)}{3 k+1}$. The initial conditions are $x(0)=\left[\begin{array}{lll}0.4 & 0.2 & 0.4\end{array}\right]^{T}$ and $\hat{x}_{i}(0)=\left[\begin{array}{lll}0 & 0 & 0\end{array}\right]^{T}(i=1,2,3,4)$. Figures $1-3$ plot the state estimation errors $x_{j}(k)-\hat{x}_{i_{j}}(k)$ $(i=1,2,3,4, j=1,2,3)$. The simulation result has confirmed the effectiveness of the distributed estimation scheme presented in this paper.

\section{Conclusions}

In this paper, we have dealt with the robust event-triggered distributed state estimation problem for a class of stochastic systems with randomly occurring uncertainties and statedependent noises over sensor networks. The randomly occurring uncertainties have been 


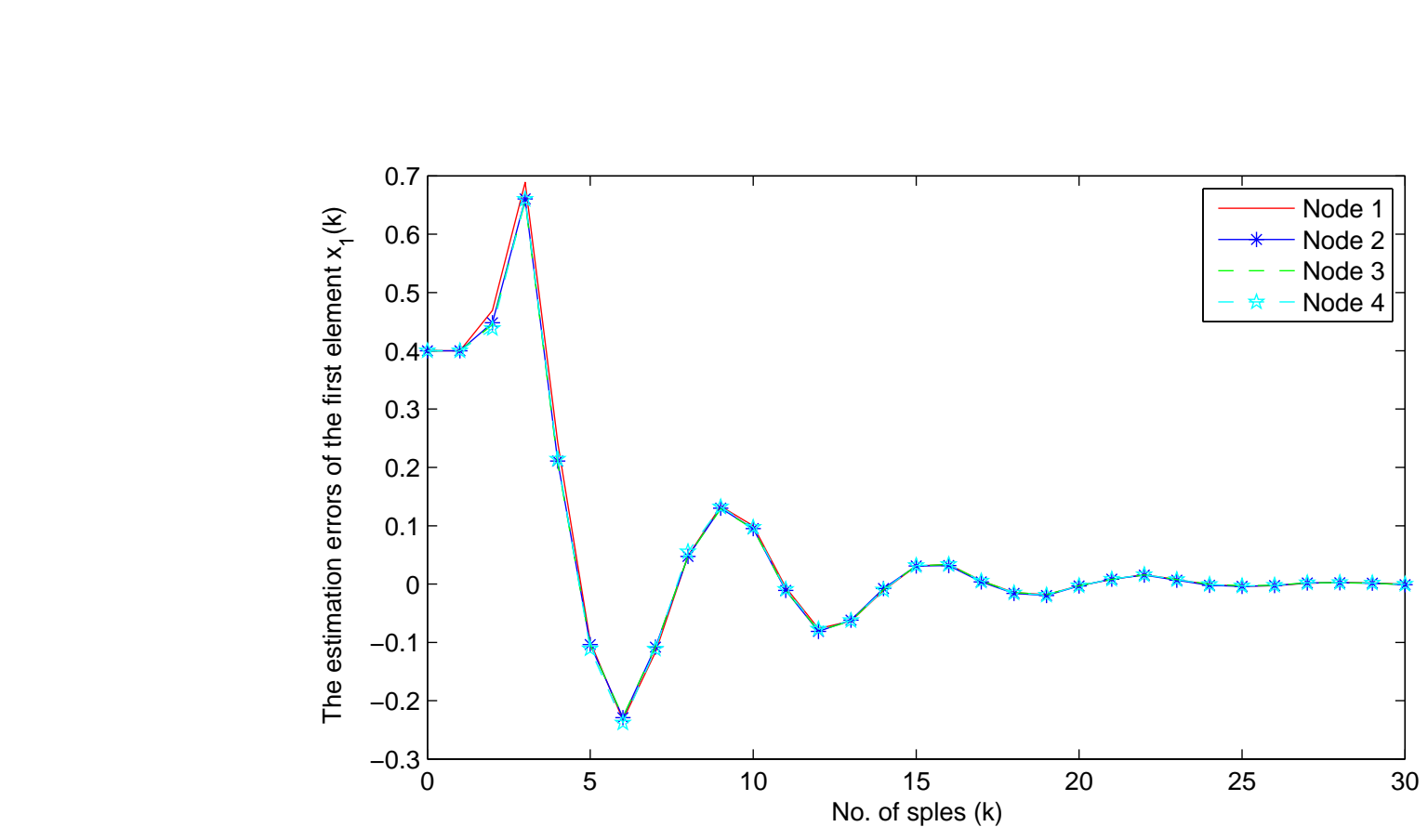

Figure 1. The estimation errors of the first element $x_{1}(k)$.

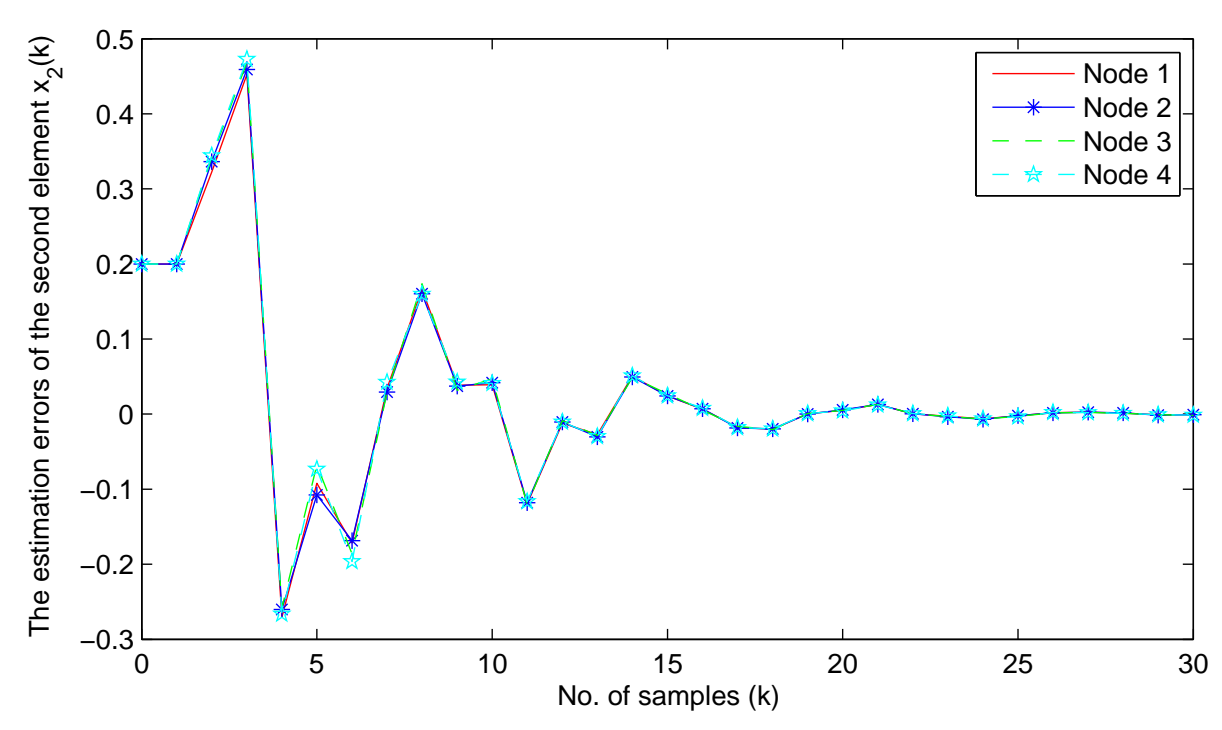

Figure 2. The estimation errors of the second element $x_{2}(k)$

modeled by the Bernoulli distributed white sequences with known conditional probabilities. An event indicator variable has been constructed and the corresponding eventtriggered scheme has been proposed to determine whether the innovation on each sensor is transmitted to the estimator or not. By employing the Lyapunov stability theorem, the distributed estimators have been designed for the dynamics of the estimation error to be exponentially mean-square stable. Finally, an illustrative example has been provided to highlight the effectiveness of the developed state estimation approach.

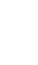




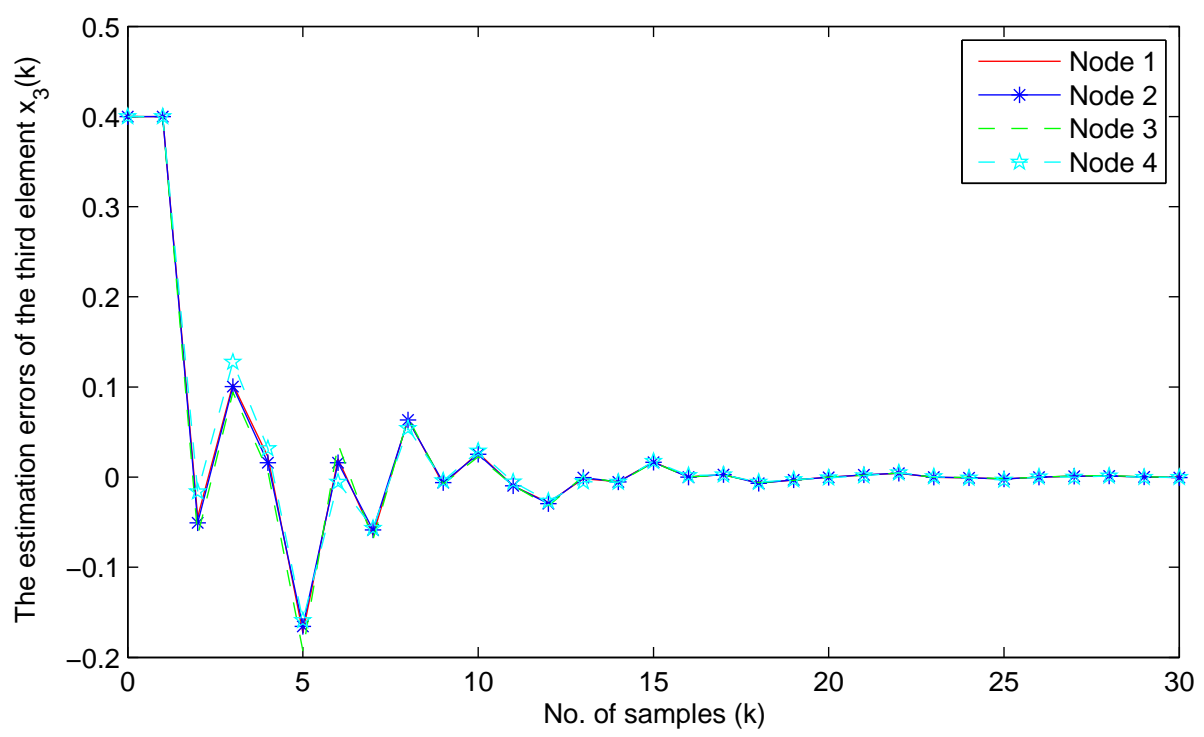

Figure 3. The estimation errors of the third element $x_{3}(k)$

\section{Acknowledgments}

This work was supported in part by the Deanship of Scientific Research (DSR) at King Abdulaziz University of Saudi Arabia under Grant 16-135-35-HiCi, the National Natural Science Foundation of China under Grants 61374127 and 61329301, the Scientific and Technology Research Foundation of Heilongjiang Education Department of China under Grant 12541061 and 12511014, and the Alexander von Humboldt Foundation of Germany.

\section{References}

Bertrand, A., and M. Moonen. 2010. "Distributed adaptive node-specific signal estimation in fully connected sensor networks-part I: sequential node updating." IEEE Trans. Signal Processing 58(10): 5277-5291.

Boyd, S., L. E. Ghaoui, E. Feron, and V. Balakrishnan. 1994. Linear Matrix Inequalities in System and Control Theory. Philadelphia: SIAM.

Cattivelli, F. S., and A. H. Sayed. 2008. "Diffusion mechanisms for fixed-point distributed Kalman smoothing." Proc. 16th European Signal Processing Conference. Lausanne, Switzerland.

Cattivelli, F. S., and A. H. Sayed. 2010. "Diffusion strategies for distributed Kalman filtering and smoothing." IEEE Trans. Automatic Control 55(9): 1520-1533.

Ding, S. X. 2013. Model-Based Fault Diagnosis Techniques-Design Schemes, Algorithms and Tools. Springer-Verlag.

Ding, S. X., P. Zhang, Ch. Chihaia, W. Li, Y. Wang, and E. L. Ding. 2008. "Advanced design scheme for fault tolerant distributed networked control systems." 17th IFAC World Congress. Seoul, Korea.

Ding, D., Z. Wang, H. Dong, and H. Shu. 2012. "Distributed $\mathcal{H}_{\infty}$ state estimation with stochastic parameters and nonlinearities through sensor networks: the finite-horizon case." Automatica 48(8): 1575-1585.

Ding, D., Z. Wang, and B. Shen. 2014. "Recent advances on distributed filtering for 
stochastic systems over sensor networks." International Journal of General Systems 43(3-4): 372-386.

Dong, H., Z. Wang, and H. Gao. 2012. "Distributed filtering for a class of time varying systems over sensor networks with quantization errors and successive packet dropouts." IEEE Trans. Signal Processing 60(6): 3164-3173.

Dong, H., Z. Wang, and H. Gao. 2013. "Distributed $\mathcal{H}_{\infty}$ filtering for a class of Markovian jump nonlinear time-delay systems over lossy sensor networks." IEEE Trans. Industrial Electronics 60(10): 4665-4672.

Donkers, M., and W. Heemels. 2012. "Output-based event-triggered control with guaranteed $\mathcal{L}_{\infty}$ gain and improved and decentralised eventtriggering." IEEE Trans. Automatic Control 57(6): 1326-1332.

Hu, J., Z. Wang, B. Shen, and H. Gao. 2013. "Quantized recursive filtering for a class of nonlinear systems with multiplicative noises and missing measurements." International Journal of Control 86(4): 650-663.

Hu, J., D. Chen, and J. Du. 2014. "State estimation for a class of discrete nonlinear systems with randomly occurring uncertainties and distributed sensor delays." International Journal of General Systems 43(3-4): 387-401.

$\mathrm{Hu}, \mathrm{S}$., and D. Yue. 2012. "Event-based $\mathcal{H}_{\infty}$ filtering for networked system with communication delay." Signal Processing 92(9): 2029-2039.

Huang, C., D. W. C. Ho, and J. Lu. 2012. "Partial-information-based distributed filtering in two-targets tracking sensor networks." IEEE Trans. Circuits and Systems I-Regular Papers 59(4): 820-832.

Lee, C., and S. Choi. 2013. "Distributed event-triggered path construction in wireless sensor networks." Journal of Wireless Networks 19(1): 1-16.

Liang, J., Z. Wang, B. Shen, and X. Liu. 2012. "Distributed state estimation in sensor networks with randomly occurring nonlinearities subject to time-delays." ACM Transactions on Sensor Networks 9(1). Art. No. 4, doi: 10.1145/2379799.2379803.

Liu, Q., Z. Wang, X. He, and D. H. Zhou. 2014. "A survey of event-based strategies on control and estimation." Systems Science and Control Engineering: An Open Access Journal 2(1): 90-97.

Li, H. 2012. "Event-triggered state estimation for a class of delayed recurrent neural networks with sampled-data information." Abstract and Applied Analysis 2012(Article ID 731453): 21 pages.

Peng, C., and T. Yang. 2013. "Event-triggered communication and $\mathcal{H}_{\infty}$ control co-design for networked control systems." Automatica 49(5): 1326-1332.

Sahoo, A., X. Hao, and S. Annathan. 2013. "Neural network-based adaptive eventtriggered control of nonlinear continuous-time systems." 2013 IEEE International Symposium on Intelligent Control 28-30. Hyderabad, India.

Shen, B., Z. Wang, and Y. S. Hung. 2010. "Distributed $\mathcal{H}_{\infty}$ - filtering in sensor networks with multiple missing measurements: the finite-horizon case." Automatica 46(10): $1682-1688$.

Shen, B., Z. Wang, Y. S. Hung, and G. Chesi. 2011. "Distributed $\mathcal{H}_{\infty}$ filtering for polynomial nonlinear stochastic systems in sensor networks." IEEE Trans. Industrial Electronics 58(5): 1971-1979.

Speranzon, A., C. Fischione, K. H. Johansson, and A. Sangiovanni-Vincentelli. 2008. "A distributed minimum variance estimator for sensor networks." IEEE Journal on Selected Areas in Communications 26(4): 609-621.

Tabuada, P.(2007). "Event-triggered real-time scheduling of stabilizing control tasks." IEEE Trans. Automatic Control 52(9): 1680-1685.

Tseng, Y. C., T. Y. Lin, Y. K. Liu, and B.-R. Lin. 2005. "Event-driven messaging services over integrated cellular and wireless sensor networks: prototyping experiences of a visitor system." IEEE Journal on Selected Areas in Communications 23(6): 1133-1145. 
Wang, Z., Y. Liu, G. Wei, and X. Liu. 2010. "A note on control of a class of discretetime stochastic systems with distributed delays and nonlinear disturbances." IEEE Trans. NanoBioscience 46(3): 543-548.

Wang, Z., F. Yang, D. W. C. Ho, and X. Liu. 2006. "Robust $\mathcal{H}_{\infty}$ filtering for stochastic time-delay systems with missing measurements." IEEE Trans. Signal Processing 54(7): 2579-2587.

Wang, Z., H. Wu, J. Liang, and X. Liu. 2013. "On modeling and state estimation for genetic regulatory networks with polytopic uncertainties." IEEE Trans. NanoBioscience 12(1): 13-20.

Zhang, X., and L. Yang. 2014. "A fiber Bragg grating quasi-distributed sensing network with a wavelength-tunable chaotic fiber laser." Systems Science and Control Engineering: An Open Access Journal 2(1): 268-274.

Zhu, W., Z.-P. Jiang, and G. Feng. 2014. "Event-based consensus of multi-agent systems with general linear models.” Automatica 50(2): 552-558. 